\title{
Query in Dealing Progressive Tricuspid Regurgitation with Mitral Valve Disease
}

\author{
Kinnaresh Baria, Jignesh Kothari, Ishan Gohil \\ Department of Cardiovascular and Thoracic Surgery, U. N. Mehta Institute of Cardiology and Research Center (Affiliated to B.J. Medical College), Ahmedabad, Gujarat, \\ India \\ ORCID \\ Kinnaresh Baria: https://orcid.org/0000-0001-8380-5514 \\ Jignesh Kothari: https://orcid.org/0000-0002-7693-2037 \\ Ishan Gohil: https://orcid.org/0000-0003-2902-6899
}

\section{Abstract}

Objective: The recommendation to repair progressive tricuspid regurgitation (TR) at the time of mitral valve (MV) surgery is questionable. We assessed the outcomes of tricuspid valve (TV) repair for progressive TR with MV surgery. Patients and Methods: We assigned 611 patients with progressive TR who had MV replacement with or without concomitant TV repair from January 2015 to December 2016. Results: There were no remarkable variation in early mortality or major morbidity rates according to the etiology of the MV disease. Median follow-up was 36 months. After adjustment for baseline characteristics using a propensity score adjustment model, there were no significant differences in the frequency of re-admission for congestive heart failure between the groups. Both groups had similar risk for other late complications affected by valve surgery such as stroke, significant hemorrhage related to anticoagulation, reoperation due to valvular heart lesions other than TV, or infective endocarditis. However, comparing the severity of TR at the last follow-up, patients with greater than progressive TR were significantly less common in the repair group of mitral regurgitation compared with those in the control group. Conclusions: The clinical benefit of simultaneous TV repair for progressive TR with MV surgery for rheumatic mitral regurgitation is certain. Concomitant TV repair cannot be routinely recommended in patients with rheumatic mitral stenosis at the time of MV repair or replacement.

Keywords: Tricuspid regurgitation, tricuspid valve, valve surgery

\section{INTRODUCTION}

Functional tricuspid regurgitation (TR) is usually seen in individuals having prolonged mitral valve (MV) disease. ${ }^{[1]}$ However, functional TR may improve after rectifying MV lesions, ${ }^{[2]}$ unaddressed significant TR is said to progress in nearly half of patients, ${ }^{[3]}$ and increase postoperative mortality and morbidity. ${ }^{[4]}$ The importance of overlooked functional TR was recently stated and numerous studies reported on simultaneous repair of functional TR. Severe TR in patients having MV disease requiring MV surgery is a definite indication (Class 1) for simultaneous tricuspid valve (TV) repair. ${ }^{[5,6]}$ Although the decision to address lesser

Received: 30-05-2021 Revised: 14-07-2021 Accepted: 18-07-2021

Published Online: 25-09-2021

\begin{tabular}{|l|l|}
\hline \multicolumn{3}{|c|}{ Access this article online } \\
\hline Quick Response Code: & Website: \\
& \\
http://www.ijcva.com
\end{tabular}

degree of functional TR during MV, surgery is not well defined (Class 2b), ${ }^{[6]}$ and the optimal strategy for the treatment of progressive TR remains controversial.

Hence, we reviewed individuals having progressive TR who needed MV surgery with or without TV repair to determine whether or not to go for TV repair in patients with MV surgery. TV repair affects the clinical and echocardiographic outcomes and to identify those patients which will be benefitted by TV repair.

Address for correspondence: Dr. Kinnaresh Baria, Department of Cardiovascular and Thoracic Surgery, U. N. Mehta Institute of Cardiology and Research Center (Affiliated to B. J. Medical College), New Civil Hospital Campus, Asarwa, Ahmedabad - 380 016, Gujarat, India. E-mail: drkinnaresh@gmail.com

This is an open access journal, and articles are distributed under the terms of the Creative Commons Attribution-NonCommercial-ShareAlike 4.0 License, which allows others to remix, tweak, and build upon the work non-commercially, as long as appropriate credit is given and the new creations are licensed under the identical terms.

For reprints contact:WKHLRPMedknow_reprints@wolterskluwer.com

How to cite this article: Baria K, Kothari J, Gohil I. Query in dealing progressive tricuspid regurgitation with mitral valve disease. Int J Cardiovasc Acad 2021;7:83-7. 


\section{Patients and Methods}

\section{Study population}

From January 2015 to December 2016, 611 patients aged $>18$ years had surgery for rheumatic MV disease at U. N. Mehta Institute of Cardiology and Research Centre [Figure 1]. Among these, seven patients expired due to cardiac complication in immediate postoperative period. Of the remaining 604 patients, 355 patients had severe mitral stenosis (MS) with progressive TR and 249 patients had severe mitral regurgitation with progressive TR on preoperative echocardiogram. One hundred and seventy-six patients underwent MV replacement with TV repair in severe MS progressive TR group, whereas 102 patients underwent MV replacement with TV repair in severe MR progressive TR group, rest patient had MV replacement only (control group). Twenty-five patients were selected in each group based on propensity score matching. Progressive TR was defined as TR with central jet $<50 \%$ of right atrium, vena contracta width $<0.7 \mathrm{~cm}$, effective regurgitant orifice $<0.40 \mathrm{~cm}^{2}$, and regurgitant volume $<45 \mathrm{ml}^{[5]}$ This study was approved by our Institutional Ethics Committee/Review Board on March 24, 2015 vide UNMICRC/2015/33, and they waived the requirement for informed patient consent because of the retrospective nature of this study.

\section{Echocardiographic evaluation}

Two-dimensional echocardiography and Doppler color-flow imaging were performed on all patients. All patients underwent transthoracic echocardiographic evaluation before operation.

The degree of TR was evaluated using the apical four-chamber view and graded as progressive TR with central jet $<50 \%$ of right atrium, vena contracta width $<0.7 \mathrm{~cm}$, effective regurgitant orifice $<0.40 \mathrm{~cm}^{2}$, and regurgitant volume $<45 \mathrm{ml}$; otherwise severe TR. ${ }^{[5]}$ The peak systolic TR jet velocity measured by continuous-wave Doppler was used to calculate the TR peak pressure gradient using the simplified Bernoulli equation (pressure gradient $=4 \times$ velocity $^{2}$ ) to estimate the peak systolic pulmonary artery pressure.

\section{Surgical procedures}

The decision to perform TV repair was affected by the degree of TR, tricuspid annular dimension, left ventricular function, combined procedures, and the presence of atrial fibrillation (AF) but was ultimately at the discretion of the attending surgeon. TV annular dimension was routinely measured pre- or intraoperatively, annular size was an indispensable indicator for combination of TV repair. In cases of TV repair, TR was repaired by Duran Ancore ring annuloplasty, and surgical techniques were determined based on each surgeon's preference.

\section{Follow-up}

Data were collected from medical charts during regular visits to the outpatient department or by telephone contact. Operative mortality was defined as death within 30 days after surgery.

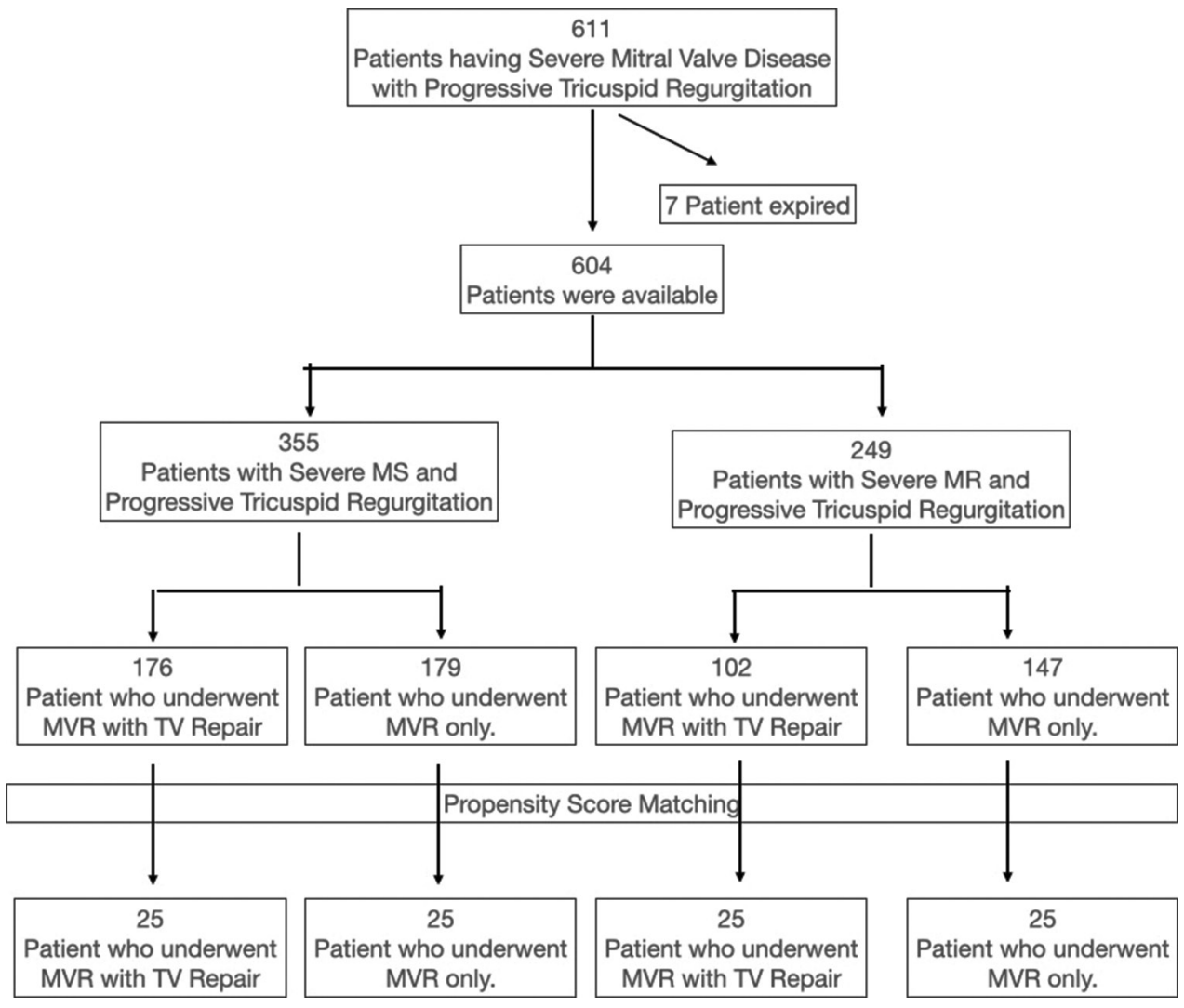

Figure 1: Flow chart of study population 
Postoperative TR grade and left ventricular ejection fraction (LVEF) were obtained from the final postoperative echocardiogram obtained after 3 months in patients who survived $>1$ months.

All patients had follow-up 3 monthly for 6 months and then 6 monthly for 3 years.

\section{Statistical analysis}

Categorical variables are presented as numbers and percentages and were compared using the Chi-squared and Fisher's exact tests. Continuous variables are expressed as mean \pm standard deviation and were compared using the $t$-test or the MannWhitney $U$-test, as appropriate. To adjust for differences in the baseline patient characteristics between groups, propensity score analysis was performed. The propensity scores were estimated without regard to outcome variables through binary logistic regression analysis incorporating 11 prespecified covariates listed in Table 1. The discrimination and calibration ability of the propensity score model was assessed using the $\mathrm{C}$ statistic and the Hosmer-Lemeshow statistic. The model was well calibrated (HosmerLemeshow test $P=0.707)$ with reasonable discrimination (C statistic: 0.880). The hazard of concomitant TV repair affecting the clinical outcomes compared with no repair was analyzed using the Cox regression model without and with adjustment by propensity scores. Cumulative incidence rates of individual and composite outcomes were also estimated using Cox regression model. Multivariable risk factors for the composite of death, TV reoperation, and congestive heart failure (CHF) requiring readmission were obtained using the Cox proportional hazards model. Variables with a $P=0.20$ on univariate analysis were candidates for the multivariable models. Multivariable analysis involved a backward elimination technique, and only variables with a $P<0.10$ were used in the final model.

To compare postoperative echocardiographic outcomes between the groups, propensity score matching was performed. In patients who had follow-up echocardiogram, propensity scores were re-estimated (HosmerLemeshow test, $P=.350$; C statistic, 0.877$)$. To develop the propensity score-matched pairs without replacement (a 1:1 match), the Greedy 5 to 1 digit match algorithm was used ${ }^{[7,8]}$ After propensity score matching, the baseline covariates were compared between the groups using the paired $t$-test or the Wilcoxon signed-rank test for continuous variables and the McNemar test or marginal homogeneity test for categorical variables. SPSS software version 24.0 (IBM) (IBM Corp. Released 2016. IBM SPSS Statistics, Version 24.0. Armonk, NY: IBM Corp.) was used for statistical analyses.

\section{RESULTS}

\section{Baseline characteristics}

Preoperative baseline patient characteristics are summarized in Table 1. Patients in the repair group had higher clinical and echocardiographic risk factors than those in the control group as evidenced by older age $(P<0.001)$, more history of previous cardiac surgery $(P<0.001)$, higher prevalence of AF $(P<0.001)$, more severe TR $(P<0.001)$, and poorer $\operatorname{LVEF}(P<0.001)$.

Two hundred forty-nine patients $(41.22 \%)$ underwent MV replacement for mitral regurgitation and 355 patients (58.7\%) for MS. There were no significant between-group differences according to the etiology of the MV disease $(P=.073)$ [Table 2].

\section{Clinical outcomes}

\section{Perioperative Results}

Cardiopulmonary bypass ( $146.3 \pm 55.9 \mathrm{~min}$ vs. $115.0 \pm 45.8 \mathrm{~min}$; $P<0.001)$ and aortic cross-clamp $(95.9 \pm 35.9 \mathrm{~min}$ vs. $73.9 \pm 33.6 \mathrm{~min} ; P<0.001)$ times were significantly longer in the repair group than in the control group. There were no significant differences in early mortality $(1.6 \%$ vs. $0.6 \%$; $P=.12)$ or major morbidity rates ( $15.5 \%$ vs. $13.4 \% ; P=.36)$.

\begin{tabular}{|c|c|c|c|}
\hline & $\begin{array}{c}\text { Repair } \\
\text { group (\%) }\end{array}$ & $\begin{array}{l}\text { No repair } \\
\text { group (\%) }\end{array}$ & $P$ \\
\hline Number of patients & 50 & 50 & \\
\hline Age (years) & $38 \pm 10.9$ & $39 \pm 10.9$ & 0.6475 \\
\hline Man & $21(42)$ & $17(34)$ & 0.5365 \\
\hline Diabetes mellitus & $4(8)$ & - & 0.1258 \\
\hline Hypertension & $4(8)$ & $3(6)$ & 1.0000 \\
\hline Chronic renal failure & $3(6)$ & & 0.2410 \\
\hline Atrial fibrillation & $6(12)$ & $8(16)$ & 0.7732 \\
\hline \multicolumn{4}{|l|}{ History of thromboembolic events } \\
\hline \multicolumn{4}{|l|}{ Stroke } \\
\hline \multicolumn{4}{|l|}{ Other thromboembolic events } \\
\hline \multicolumn{4}{|l|}{ Mitral valve pathology } \\
\hline $\begin{array}{l}\text { Predominantly mitral } \\
\text { regurgitation }\end{array}$ & $25(50)$ & $25(50)$ & 0.8415 \\
\hline Predominantly mitral stenosis & $25(50)$ & $25(50)$ & 0.8415 \\
\hline \multicolumn{4}{|l|}{ Tricuspid regurgitation } \\
\hline \multicolumn{4}{|l|}{ Mild } \\
\hline Progressive & 50 & 50 & \\
\hline $\operatorname{LVEF}(\%)<40 \%$ & $1(1)$ & 0 & 1.000 \\
\hline Surgery type-MVR & 50 & 50 & \\
\hline
\end{tabular}

LVEF: Left ventricular ejection fraction, MVR: Mitral valve repair

Table 2: Operative outcomes

\begin{tabular}{lccc}
\hline & $\begin{array}{c}\text { Repair } \\
\text { group (\%) }\end{array}$ & $\begin{array}{c}\text { No repair } \\
\text { group (\%) }\end{array}$ & $\boldsymbol{P}$ \\
\hline Operative mortality & $4(8)$ & $3(6)$ & 1.000 \\
Early complications & & & \\
$\quad$ Sternal bleeding & $5(10)$ & $2(4)$ & 0.4331 \\
Pericardial effusion & $4(8)$ & $2(4)$ & 0.6737 \\
Wound problem & - & - & \\
Permanent pacemaker insertion & $1(2)$ & - & 1.000 \\
Low cardiac output syndrome & $2(4)$ & - & 0.4751 \\
Requirement for dialysis & $3(6)$ & - & 0.2410 \\
Mediastinitis & - & - & \\
Neurologic complication & $1(2)$ & - & 1.0000 \\
\hline
\end{tabular}




\section{Primary and secondary end points}

During a median clinical follow-up period of 36 months, there were seven early deaths, two readmissions for CHF. There were no significant differences in the frequency of re-admission for CHF between the groups. Both groups had similar risk for other late complications affected by valve surgery such as stroke, significant hemorrhage related to anticoagulation, reoperation due to valvular heart lesions other than $\mathrm{TV}$, or infective endocarditis.

\section{Comparison of echocardiographic outcomes between the} two groups: propensity score matching

Of 100 participants, $>3$ months after surgery had follow-up echocardiogram for $>6$ months. Median follow-up duration was 36 months. Propensity score matching for the 604 patients yielded 100 matched pairs of patients. In the matched cohort, there were no significant between- group differences for any baseline covariates.

Preoperative and postoperative TR grades were compared between the 2 groups in the matched cohort. There were no between-group differences in preoperative TR grades $(P>0.99)$; however, comparing the severity of TR at the last follow-up, patients with greater than progressive TR were significantly less common in the repair group of mitral regurgitation compared with those in the control group.

\section{Discussion}

Several studies have shown that uncorrected significant functional TR after left heart valve surgery is associated with increased mortality and morbidity ${ }^{[4,9-11]}$ and decreased functional outcome. ${ }^{[9,12-14]}$ However, data are limited with regard to whether uncorrected functional TR will progress despite resolution of the left heart lesion responsible for overloading the right ventricle. Updated guidelines of the American College of Cardiology/American Heart Association regarding valve disease suggest that TV repair is beneficial for severe TR in patients with MV disease requiring MV surgery (Class 1, level of evidence: B-NR). ${ }^{[5]}$ According to these guidelines, however, less than severe TR in patients undergoing MV surgery is not a definite indication for tricuspid annuloplasty (Class IIa, level of evidence: B-NR). The European Society of Cardiology Guidelines on the management of valvular heart disease also state that severe TR in a patient undergoing left-sided valve surgery is the only definite indication for TV repair (Class I, level of evidence: C). ${ }^{[6]}$

Recently, as the significance of TR has been addressed, a few studies have reported on mild-to-moderate TR. Song et al. ${ }^{[14]}$ reported that $7.7 \%$ of untreated mild TR progressed to late significant TR despite successful left-sided valve surgery through a retrospective study involving 638 patients during a mean follow-up period of 64 months. Because late significant TR was associated with worse clinical outcomes, they suggested that aggressive surgical intervention may be considered in patients with risk factors for developing late significant TR. Another retrospective comparison of surgical and conservative treatment for mild-to-moderate functional TR showed that concomitant TV repair with MV repair or replacement decreased right ventricular diameter and the average grade of TR. ${ }^{[15]} \mathrm{Kim}$ et al.$^{[16]}$ reported a retrospective study of 236 patients with mild-to-moderate functional TR who underwent MV replacement for rheumatic MV disease. In their study, concomitant TV repair for mild-to- moderate functional TR was associated with better postoperative TV function.

Dreyfus et al.${ }^{[3]}$ recommended that the TV annulus should be measured at the time of left heart valve surgery and tricuspid dilatation should be corrected regardless of the severity of TR because TV disease is correlated with tricuspid dilatation rather than the degree of TR. They measured the tricuspid annular diameter intraoperatively from the anteroseptal commissure to the anteroposterior commissure. Patients with a tricuspid annular dimension $>70 \mathrm{~mm}$ underwent tricuspid annuloplasty, which improved functional status.

Yilmaz et al. ${ }^{[17]}$ analyzed changes in the degree of functional TR in 699 patients who underwent MV repair for degenerative MV disease. Their data demonstrated that the progression of TR in patients undergoing MV repair is unusual, and that coexistent TR did not affect late mortality. The authors emphasized the significance of preoperative comorbidities in the determination of clinical outcomes rather than the presence of functional TR.

In our study, concomitant TV repair was not associated with better clinical outcomes, in patients with severe MS but did result in better TR grades. These results are not much different from other retrospective studies, although the size of our study was smaller and the disease entity was extended to rheumatic MV disease. In other words, better TV function did not seem to affect long-term clinical outcomes in this study. However, considering that it takes longer time for significant TR to affect heart function, compared with the clinical course of left sided valve disease; the clinical follow-up period of this study may not be long enough to verify the efficacy of concomitant TV repair.

\section{Limitations}

Our study was retrospective in nature and nonrandomized. Moreover, surgical indications and surgical techniques were not uniform among surgeons who did or did not perform concomitant TV repair. Rigorous statistical adjustment was used for analysis. Echocardiographic values that are associated with the right heart, such as tricuspid annular dimension, TV tethering distance, and TV tethering area could not be routinely estimated, and right ventricular function was not quantified. The median follow-up period of this study was 36 months (i.e. 3 years), which may not be long enough to interpret the effect of significant TR on clinical outcomes.

\section{Conclusions}

The clinical benefit of concomitant TV repair for moderate concomitant functional TR during MV surgery for rheumatic 
mitral regurgitation is certain. Concomitant TV repair cannot be routinely recommended in patients with rheumatic MS at the time of MV repair or replacement.

\section{Financial support and sponsorship}

This work was supported by U. N. Mehta Institute of Cardiology and Research Center itself and received no specific grant from any funding agency, commercial, or not-for-profit sectors.

\section{Conflicts of interest}

There are no conflicts of interest.

\section{References}

1. Sagie A, Freitas N, Chen MH, Marshall JE, Weyman AE, Levine RA. Echocardiographic assessment of mitral stenosis and its associated valvular lesions in 205 patients and lack of association with mitral valve prolapse. J Am Soc Echocardiogr 1997;10:141-8.

2. Braunwald NS, Ross J Jr., Morrow AG. Conservative management of tricuspid regurgitation in patients undergoing mitral valve replacement. Circulation 1967;35:163-9.

3. Dreyfus GD, Corbi PJ, Chan KM, Bahrami T. Secondary tricuspid regurgitation or dilatation: Which should be the criteria for surgical repair? Ann Thorac Surg 2005;79:127-32.

4. Kuwaki K, Morishita K, Tsukamoto M, Abe T. Tricuspid valve surgery for functional tricuspid valve regurgitation associated with left-sided valvular disease. Eur J Cardiothorac Surg 2001;20:577-82.

5. Otto CM, Nishimura RA, Bonow RO, Carabello BA, Erwin JP $3^{\text {rd }}$, Gentile F, et al. 2020 ACC/AHA guideline for the management of patients with valvular heart disease. A report of the American College of Cardiology/American Heart Association Joint Committee on Clinical Practice Guidelines. Circulation 2021;143:e35-71.

6. Baumgartner H, Falk V, Bax JJ, De Bonis M, Hamm C, Holm PJ, et al. 2017 ESC/EACTS Guidelines for the management of valvular heart disease. The task force for the management of valvular Heart disease of the European Society of Cardiology and the European Association for
Cardio-Thoracic Surgery. Eur Heart J 2017;38:2739-91.

7. Gum PA, Thamilarasan M, Watanabe J, Blackstone EH, Lauer MS Aspirin use and all-cause mortality among patients being evaluated for known or suspected coronary artery disease: A propensity analysis. JAMA 2001;286:1187-94.

8. Sagie A, Schwammenthal E, Newell JB, Harrell L, Joziatis TB, Weyman AE, et al. Significant tricuspid regurgitation is a marker for adverse outcome in patients undergoing percutaneous balloon mitral valvuloplasty. J Am Coll Cardiol 1994;24:696-702.

9. McCarthy PM, Bhudia SK, Rajeswaran J, Hoercher KJ, Lytle BW, Cosgrove DM, et al. Tricuspid valve repair: Durability and risk factors for failure. J Thorac Cardiovasc Surg 2004;127:674-85.

10. Hornick P, Harris PA, Taylor KM. Tricuspid valve replacement subsequent to previous open heart surgery. J Heart Valve Dis 1996;5:20-5.

11. Groves PH, Ikram S, Ingold U, Hall RJ. Tricuspid regurgitation following mitral valve replacement: An echocardiographic study. J Heart Valve Dis 1993;2:273-8.

12. Nath J, Foster E, Heidenreich PA. Impact of tricuspid regurgitation on long-term survival. J Am Coll Cardiol 2004;43:405-9.

13. Groves PH, Lewis NP, Ikram S, Maire R, Hall RJ. Reduced exercise capacity in patients with tricuspid regurgitation after successful mitral valve replacement for rheumatic mitral valve disease. Br Heart J 1991;66:295-301.

14. Song H, Kim MJ, Chung CH, Choo SJ, Song MG, Song JM, et al. Factors associated with development of late significant tricuspid regurgitation after successful left-sided valve surgery. Heart 2009;95:931-6.

15. Smíd M, Cech J, Rokyta R, Roucka P, Hájek T. Mild to moderate functional tricuspid regurgitation: Retrospective comparison of surgical and conservative treatment. Cardiol Res Pract 2010;2010:143878.

16. Kim JB, Yoo DG, Kim GS, Song H, Jung SH, Choo SJ, et al. Mild-to-moderate functional tricuspid regurgitation in patients undergoing valve replacement for rheumatic mitral disease: The influence of tricuspid valve repair on clinical and echocardiographic outcomes. Heart 2012;98:24-30.

17. Yilmaz O, Suri RM, Dearani JA, Sundt TM $3^{\text {rd }}$, Daly RC, Burkhart HM, et al. Functional tricuspid regurgitation at the time of mitral valve repair for degenerative leaflet prolapse: The case for a selective approach. J Thorac Cardiovasc Surg 2011;142:608-13. 